International Journal of Engineering \& Technology, $7(1.5)(2018) 122-125$
International Journal of Engineering \& Technology
SPC
Website: www.sciencepubco.com/index.php/IJET
Research paper

\title{
EMD based Hilbert energy spectrum analysis of adventitious lung wave signals
}

\author{
Shankar B. B. ${ }^{1 *}$, D. Jayadevappa ${ }^{2}$ \\ ${ }^{1}$ Research Scholar, Jain University, Bangalore, India \\ ${ }^{2}$ Dept. of IT, JSSATE, Bangalore, India \\ *Corresponding author E-mail: sankrubb@gmail.com
}

\begin{abstract}
The respiratory adventitious waves are analyzed effectively by time frequency analysis. In this paper, we present a new approach for rectifying the abnormality in adventitious wave. Basically, there are two types of respiratory sound waves and these are classified as wheezes and crackles. The proposed method utilizes the time frequency analysis using spectrum analysis method. The modified Empirical Mode Decomposition (EMD) called Ensemble Empirical Mode Decomposition (EEMD) to plot energy spectrum of adventitious wave is used in this work. The proposed method decomposes the respiratory adventitious wave into a different Intrinsic Mode Function (IMF). The long and short duration adventitious waves are present in a wheezing subject and this leads to production of non stationary and nonlinear sound waves. The empirical mode decomposition (EMD) decomposes such characteristic waves. The available spectrogram analyzes techniques related to additive expansions and explore amplitude wise time-frequency distribution. The methodology discussed in this context responding greatly even for correlative noise and explores energy spectra in addition to amplitude spectra. The various IMFs such produced are exhibits the fine details of adventitious wave and thus pattern can be predicted for final residual. The energy spectrum can be viewed as a diagnostic tool for accurate analysis of wheezing pattern. The decomposed frequency patterns indicate the physiological aspects. The instantaneous frequency and Hilbert energy spectrum based on above mentioned a method are employed by IMF to analyze and present the result in time-frequency distribution to explore the characteristics of inherent properties adventitious signals. The Hilbert marginal spectrum has been used to indicate overall energy distribution from each frequency component. Finally, the resultant EMD analysis along with EEMD energy spectrum is better for asthmatic subject and solves mode mixing problems.
\end{abstract}

\section{Introduction}

The Hilbert-Huang transform (HHT) is a technique which is a super composition of the empirical mode decomposition (EMD) and the Hilbert spectral analysis (HSA), to provide a powerful technique for analysing nonlinear and non-stationary wave data. From Taplidou's era [12] time -frequency-based analysis of lung sounds have been designed. The robustness of various adventitious waves well analysed and presented [13]. The primary goal of HHT to decompose the wheezing wave and also normal respiratory wave with the combination of EMD and Hilbert spectral analysis. Wheezing can be heard usually at all over the chest of patients with normal respiratory function. The abnormal irregular, musical sound signals produced by human lungs and airways called adventitious respiratory signals. There are fine and coarse crackles, wheezes and stirdor. The discontinuous adventitious sounds (DAS) are short, popping sound signals. Crackles or rales are the examples for DAS. The rales are generated at the level of alveoli, 'Rales in the tails'. The continuous adventitious sounds (CAS) are low or high-pitched sound signals produced during expiration stage of respiration. The wheezing or rhonchi are the types of CAS. The Rhonchi are generated at the level of bronchi, 'Rhonchi in the bronchi'. The Hilbert - Huang Transformation is the very effective frequency domain analysis technique for adventitious sounds which are nonperiodic and nonstationary signals. The inbuilt components of adventitious sound waves must be split and analysed accurately with time-frequency analysis. The non-stationary signal analysis like wavelet and spectrogram, HHT algorithm is used for satisfactory accurate results. The Hilbert energy spectrum also plotted for better analysis. The overview of adventitious sound in terms of spectral changes in power over time is achieved by wavelet transform which gives a perfect balance between temporal and frequency precision. The wavelet transform is not a suitable technique for extracting the oscillatory-like features from the adventitious data, in such situation empirical mode decomposition (EMD) of Hilbert-Huang Transform (HHT) which is iterative procedure finds the most promising technique. It provides the specific level of frequencies range that cannot be predicted in advance by us. These frequencies data have a lot of power and are nearly half of the previous frequency that was extracted. The specialized technique which takes care of fine information embedded inside an adventitious sound must be analysed in terms Intrinsic Mode Function (IMF) which is a basic building block of EMD. The EMD and Ensemble EMD (EEMD) based Hilbert spectrum is a helpful technique to establish CAS more accurately than another conventional time-frequency analysis [3]. The EMD and EEMD stand at the tip of the edge of time-frequency analysis technique compare to STFT, wavelets and any form of Fourier 
Transformation technique. Sinusoids decomposition of Fourier domain gives unsatisfactory information and sidelobesartifacts. The proposed system finds the envelopes between minima and maxima along with residuals and IMFs components. It finds a suitable position among other adventitious sound analysis technique and at the same time as it is a specialized technique consumes more time duration to compute the entire 10-second duration wave. However, the sound wave of a certain pitch duration is considered to avoid delay. The enhanced EMD and EEMD ensembles with zero noise level are achieved.

\section{Materials and method}

The adventitious lung sounds are available online at http://www.easyauscultation.com/adventitious-lung-sounds have been utilized for the study. The brief description about the different crackles and wheezes also provided in this dataset. The sound waves are for different stages of continuous and discontinuous adventitious sound and are usually quasi-periodic. The waves so collected are having total wave duration of 20 seconds and of 160000 samples with the sampling rate of 8000 Hz. The amplitude range lies in between $-110 \mathrm{~dB}$ and $+110 \mathrm{~dB}$ and is obtained by a single channel. These are sufficient for the proposed study and are enlarged using MATLAB for discussion.

\subsection{Empirical Mode Decomposition}

EMD is a sub-technique in HHT to exhibit time-frequency components of nonlinear and nonstationary wave signals. This technique decomposes a various set of functions in terms of the specialized set of functions called intrinsic mode functions (IMFs). The technique uses an iterative sifter. The various steps involved in decomposition process is as follow.

i) Identifying maxima and minima of wheezing wave.

ii) Interpolate the maxima and minima of this wheezing wave.

iii) Indicate the upper envelope by connecting all maxima peaks.

iv) Indicate the lower envelope by connecting all minima peaks.

v) Illustrate the local mean.

vi) Iterate the final residual.

Thus, the IMF function for wave signal analytically represented by:

$$
Z(t)=X(t)+j H\{X(t)\}=X(t)+j Y(t)=a(t) \exp \left(j \int \omega(t) d t\right)
$$
where

$$
\begin{aligned}
& a(t)=\sqrt{X^{2}(t)+Y^{2}(t)} \\
& \omega(t)=\frac{d}{d t}\left\{\tan ^{-1}\left(\frac{Y(t)}{X(t)}\right)\right\}
\end{aligned}
$$

The amplitude spectrum and energy spectrum based on the above equation is plotted for all instantaneous wheezing wave.Hilbert energy and amplitude spectrum of the set of various IMFs are plotted for EMD with Gaussian noise [8]. Thus, this spectral analysis gives the higher time and frequency resolution.

B. Hilbert spectrum and marginal spectrum.

Hilbert spectrum $\mathrm{H}\left({ }^{\omega}\right.$,t) is specified by.

$$
H(\omega, t)=\operatorname{Re} \sum_{i=1}^{n} a_{i}(t) e^{j \int \omega_{i}(t) d t}
$$

Where Re[] symbolizes the real part of equation within brackets. The marginal spectrum, $\mathrm{h}(\omega)$, can be determined as:

$$
h(\omega)=\int_{0}^{T} H(\omega, t) d t
$$

Here $\mathrm{T}$ indicates the wheezing wave length. The Hilbert spectral analysis provides the variation of magnitude for every timefrequency component and the marginal spectral analysis provides the variation of the complete amplitude for each and every frequency component. Consequently, local marginal spectral analysis provides the variation of the total amplitude for frequency of each IMF component, which is given as

$$
h_{i}(\omega)=\int_{0}^{T} H_{i}(\omega, t) d t
$$

\section{Results}

The different values result in the various number of IMFs and number of iterations are required to the computation of entire process. Threshold numbers after optimization are still under investigation to explore the distribution of several IMFs with the sifter process [7]. Every IMF distribution is estimated with a mathematical equation with the same number of upper and lower envelopes and at the most may vary by maximum one envelope either in the up or below-called minima. The EMD results in exploring the various number of IMFs and at the end one residue wave. The initial IMFs exhibit the lower order IMFs which are due to rapid oscillations while the end IMFs exhibit higher order IMFs which are due to low oscillations. The general trend of the time series is revealed by the final IMF wave called residue.

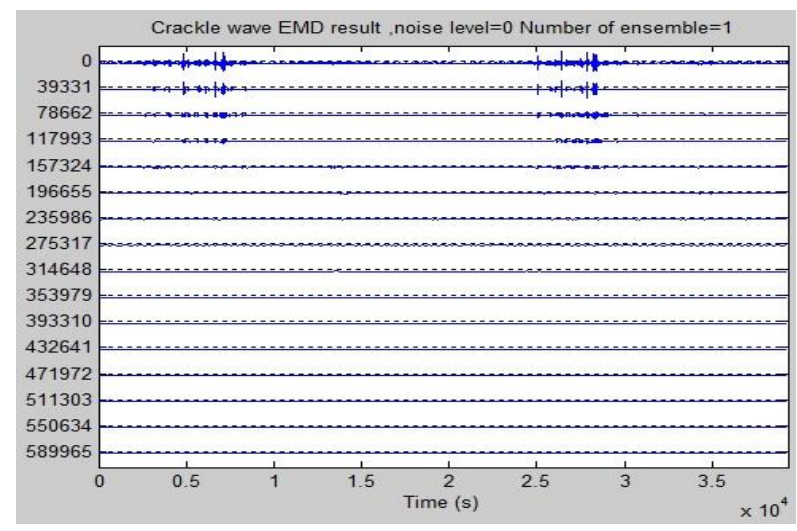

Fig.1: The EMD IMFs of the correlativeDASRSW

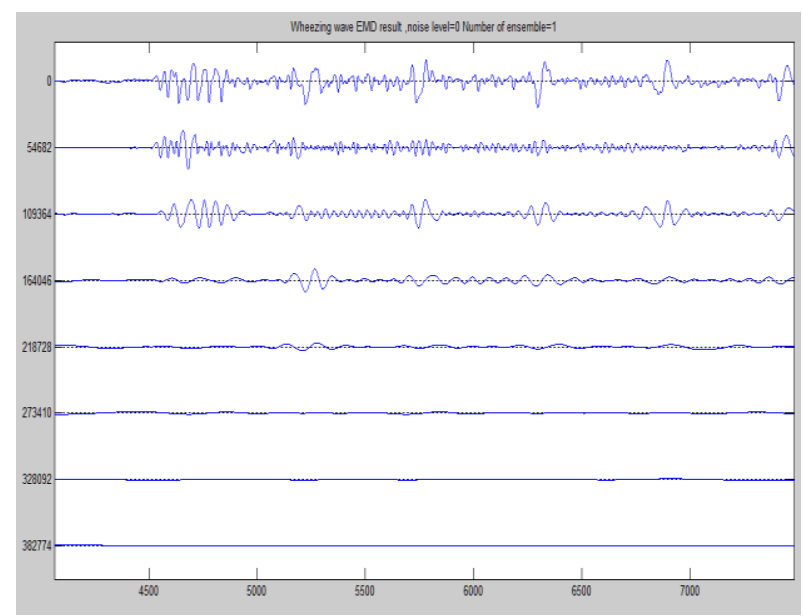

Fig. 2: Wheezing sound showing EEMD

The same adventitious waveform of continuous and discontinuous type wave is analyzed using both the techniques Empirical Mode Decomposition and Ensemble Empirical Mode Decomposition, the original adventitious waveform is same, but the final outputs are different. Total, 14 IMF's along with residual are calculated IMF'S has been calculated and shown in fig.1. The first step is to set the initial value equal to the original adventitious sound and now extrema of the adventitious sound can be found. Interconnect all the minima with spline functions to form the lower envelope. 
Now mean and standard deviation can be calculated for both the envelope. Finally, subtract this mean from the input wave. Residual sound wave is calculated and displayed at the end. Fig.2 shows the EEMD of wheezing sound waves in terms of various IMFs exhibiting true IMF set [7]. The Hilbert energy spectrum plotted below shows the wheezing wave energy distribution in a continuous limited wave signal. The continuous high frequency components at regular interval can be viewed by the tool shown in fig.3. The discontinuous high frequency components can be viewed in the fig. 4 which clearly shows the crackle wave at different interval of time.

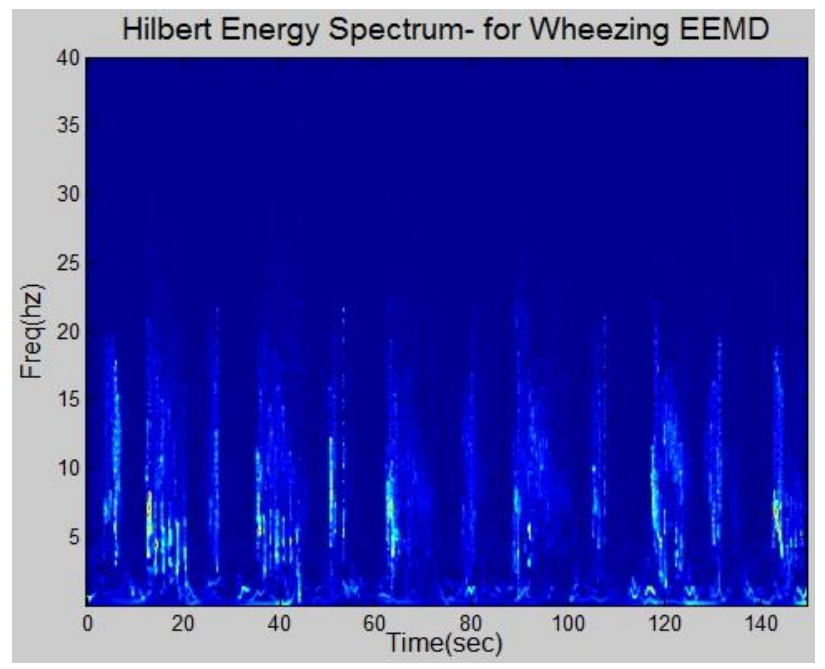

Fig. 3: The Hilbert energy spectrum with EEMD for CASRSW

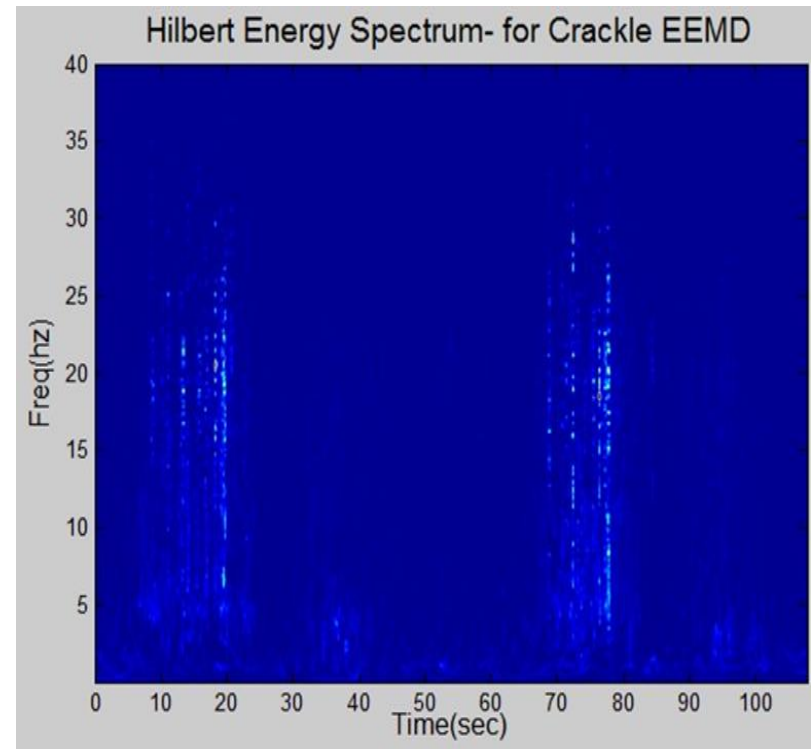

Fig. 4: The Hilbert energy spectrum with EEMD for DASRSW

The below-mentioned notation is used to identify the complex equation of basic harmonic wave [6].

$\exp (j \beta t)=\cos (\beta t)+j \sin (\beta t)$

The Equation gives bases for analytic wheezing waveform. The marginal time-frequency distribution is the important plot in order to analyse the strength of the wheezing wave after plotting energy spectrum for wheezing and crackle wave shown in fig. 3 and fig. 4 respectively.

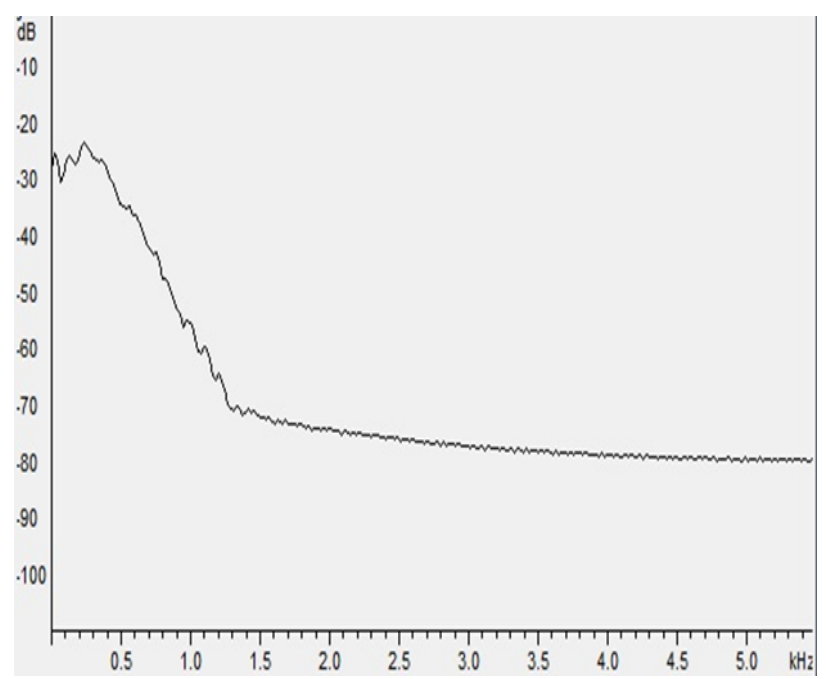

Fig. 5: The marginal frequency distribution of crackle wave at the different stages

The marginal frequency distribution of short adventitious wave is plotted in the fig. 5. The statistical calculation of short crackle wave is calculated and tabulated below.

\begin{tabular}{|lll}
\hline Column 0 & mean: 316.318830 & sd: 116.467019 \\
Column 1 & mean: 848.776372 & sd: 346.492988 \\
Column 2 & mean: 2591.745202 & sd: 361.313958 \\
Column 3 & mean: 3776.036850 & sd: 431.216165 \\
- & & \\
Statistics of CASRSW computed between 0.0 and 2.809251700680272 \\
$(\mathrm{n}=276) \mid$
\end{tabular}

\section{Discussion and conclusions}

The ultimate goal of EMD and EEMD spectrum analysis is to decompose the adventitious waves in absence of mode mixing. EEMD utilizes the statistical value of the noise mixed with the adventitious wave, but the main issue here is how to treat the multimedia distribution of the IMFs. The difference between nonlinear multiplicative interactions and iterations using both EMD and EEMD spectral analysis were discussed. The Fourier analysis for additive noise or Wavelets for correlative noise exhibited ambiguity which has to be recalculated to find additive or multiplicative [7]. The procedure is totally independent whether the adventitious wave is continuous or discontinuous as this method is nonlinear and non-stationary and which is adaptive in nature. The reconstruction of CASRSW and DASRSW are extremely easy. However, EEMD is a bit time consuming compared to EMD is noted. Interpretation of the fine information in an adventitious wave of each IMF is a really exciting task.

The present study has strongly suggested that comparative and conclusive aspects of continuous and discontinuous adventitious waves are comparatively many similar but the variations in different pitch of waves with energy and amplitude gives better pictorial representation. The addition of Gaussian noise sets a global reference for EMD and EEMD procedure. Finally, the advancement in an adventitious sound analysis, spectral EEMD became a standard procedure over EMD.

\section{References}

[1] Lozano, Manuel, José Antonio Fiz, and Raimon Jané. "Performance Evaluation of the Hilbert-Huang Transform for Respiratory Sound Analysis and Its Application to Continuous Adventitious Sound Characterization." Signal Processing 120 (2016): 99-116. Web. 
[2] Colominas, Marcelo A., Gastón Schlotthauer, and María E. Torres. "Improved Complete Ensemble EMD: A Suitable Tool for Biomedical Signal Processing." Biomedical Signal Processing and Control 14 (2014): 19-29. Web

[3] Wu, Zhaohua, and Norden E. Huang. "Ensemble Empirical Mode Decomposition: A Noise-Assisted Data Analysis Method." Advances in Adaptive Data Analysis 01.01 (2009): 1-41. Web.

[4] Tabloid, S. A., \& Hadjileontiadis, L. J. (2007). Wheeze detection based on time-frequency analysis of breath sounds. Computers in Biology and Medicine, 37 (8), 1073-1083. doi:10.1016/j. compbiomed.2006.09.007

[5] Yamashita, M., Himeshima, M., \& Matsunaga, S. (2014). Robust classification between normal and abnormal lung sounds using adventitious-sound and heart-sound models. 2014 IEEE International Conference on Acoustics, Speech and Signal Processing (ICASSP). Do: 10.1109/icassp. 2014.6854437

[6] Huang, N. E., Hu, K., Yang, A. C., Chang, H., Jia, D., Liang, W.Wu, Z. (2016). On Holo-Hilbert spectral analysis: A full informational spectral representation for nonlinear and nonstationary data. Philosophical Transactions of the Royal Society A Mathematical, Physical and Engineering Sciences, 374 (2065), 20150206. Do: 10.1098/rsta. 2015.0206

[7] Karagiannis, A.; Constantinou, P.,; "Investigating performance of Empirical Mode Decomposition application on electrocardiogam," Biomedical Engineering Conference (CIBEC), 2010th Cairo International, vol., no., 1 4Dec. 2010 doi:CIBEC.2010.5716048

[8] T. Padmapriya and V. Saminadan, "Priority based fair resource allocation and Admission Control Technique for Multi-user Multiclass downlink Traffic in LTE-Advanced Networks", International Journal of Advanced Research, vol.5, no.1, pp.1633-1641, January 2017.

[9] S.V.Manikanthan and K.srividhya "An Android based secure access control using ARM and cloud computing", Published in: Electronics and Communication Systems (ICECS), 2015 2nd International Conference on 26-27 Feb. 2015,Publisher: IEEE,DOI: 10.1109/ECS.2015.7124833.

[10] M. Rajesh, Manikanthan, "GET-UP-AND-GO EFFICIENT MEMETIC ALGORITHM BASED AMALGAM ROUTING PROTOCOL", International Journal of Pure and Applied Mathematics, ISSN NO:1314-3395, Vol-116, No. 21, Oct 2017.

[11] Rajesh, M., and J. M. Gnanasekar. \&quot;Path observation-based physical routing protocol for wireless ad hoc networks.\&quot; International Journal of Wireless and Mobile Computing 11.3 (2016): 244-257. 